\title{
Successful Treatment with Steroid Pulse Therapy for Secondary Organizing Pneumonia with Respiratory Failure due to COVID-19: A Single-Center Case Series
}

Kentaro Tamura ${ }^{1}$, Saiko Nishioka ${ }^{1}$, Nobumasa Tamura ${ }^{1}$, Zenya Saito ${ }^{1}$, and Kazuyoshi Kuwano $^{2}$

${ }^{1}$ Atsugi City Hospital

${ }^{2}$ Jikei University School of Medicine

July 2, 2020

\begin{abstract}
This is a first observational study of four patients on steroid pulse therapy for secondary organizing pneumonia with respiratory failure due to the Coronavirus Disease 2019 (COVID-19) in our hospital. All patients needed invasive mechanical ventilation and had bimodal worseness of their respiratory status with organizing pneumonia after intubation. All cases could successfully discontinue oxygen therapy without any severe adverse events after this pulse therapy. Although few previous reports showed the efficacy and safety of this therapy, this therapy is believed to be effective on some optimal patients. Hence, further studies to explore this efficacy and safety were needed.
\end{abstract}

Title

Successful Treatment with Steroid Pulse Therapy for Secondary Organizing Pneumonia with Respiratory Failure due to COVID-19: A Single-Center Case Series

\section{Short running title}

Steroid for SOP due to COVID-19

\section{Authors}

Kentaro Tamura ${ }^{1,2}$, Saiko Nishioka ${ }^{1,2}$, Nobumasa Tamura ${ }^{1,2}$, Zenya Saito ${ }^{1,2}$, Kazuyoshi Kuwano ${ }^{2}$

\section{Affiliations}

Division of Respiratory Diseases, Department of Internal Medicine, Atsugi City Hospital, Kanagawa, Japan Address: 1-16-36 Mizuhiki, Atsugi, Kanagawa, 243-8538, Japan

Division of Respiratory Diseases, Department of Internal Medicine, The Jikei University School of Medicine, Tokyo, Japan

Address: 3-25-8 Nishi-Shimbashi, Minato, Tokyo, 105-8461, Japan

\section{Corresponding Author}

Kentaro Tamura

Division of Respiratory Diseases, Department of Internal Medicine, Atsugi City Hospital, Kanagawa, Japan 
Address: 1-16-36 Mizuhiki, Atsugi, Kanagawa, 243-8538, Japan Tel: +81-46-221-1570 Fax: +81-46-294-3335 E-mail:kentaro.tamura.512@gmail.com

Division of Respiratory Diseases, Department of Internal Medicine, The Jikei University School of Medicine, Tokyo, Japan

Address: 3-25-8 Nishi-Shimbashi, Minato, Tokyo, 105-8461, Japan

\begin{abstract}
This is a first observational study of four patients on steroid pulse therapy for secondary organizing pneumonia with respiratory failure due to the Coronavirus Disease 2019 (COVID-19) in our hospital. All patients needed invasive mechanical ventilation and had bimodal worseness of their respiratory status with organizing pneumonia after intubation. All cases could successfully discontinue oxygen therapy without any severe adverse events after this pulse therapy. Although few previous reports showed the efficacy and safety of this therapy, this therapy is believed to be effective on some optimal patients. Hence, further studies to explore this efficacy and safety were needed.
\end{abstract}

\title{
Keywords
}

COVID-19, Secondary organizing pneumonia, Steroid, Steroid pulse therapy

\section{Main text}

\section{Background}

Coronavirus disease 2019 (COVID-19) has been spreading worldwide since the first outbreak in China, in December 2019. The Radiological Society of North America reported that consolidation, reserve halo sign, and other findings of organizing pneumonia were found in the late phase of COVID-19 ${ }^{1}$. In general, the treatment for secondary organizing pneumonia (SOP) is similar to that for cryptogenic organizing pneumonia (COP). The British Thoracic Society guidelines suggested a steroid pulse therapy (SPT) for some cases with fulminant $\mathrm{COP}^{2}$. On the other hand, a previous report showed that systemic corticosteroids delayed viral shedding and that they were harmful for other coronaviruses ${ }^{3}$. Therefore, a routine use of systemic corticosteroids is not recommended for viral infections at this time. However, some reports suggested that the steroid therapy might suppress a cytokine storm by COVID- $19^{4}$. One retrospective study showed that the treatment with methylprednisolone for COVID-19 reduced mortality in some cases ${ }^{4}$. SPT was assumed to be effective in the late phase for SOP with respiratory failure. This study aimed to investigate the efficacy and safety of SPT for patients with SOP and respiratory failure due to COVID-19.

Methods

This is a single-center retrospective observational study conducted at Atsugi City Hospital, Kanagawa, Japan. This study was approved by the ethics committee of this hospital (approval number: R2-07). Data among adult inpatients ([?]20 years old) with COVID-19 treated with SPT from February 5 to May 31, 2020, were retrospectively reviewed. Throat or nasal swabs were taken for the reverse transcription polymerase chain reaction (RT-PCR) test. A diagnosis of COVID-19 was determined when both clinical symptoms and positive RT-PCR were observed. Day 1 was defined as the first onset of the symptoms.

\section{Results}

Four cases of about 30 adult inpatients with COVID-19 were treated with SPT.

\section{Case 1}

This is the case of a 61-year-old man with hypertension and who is an ex-smoker, who presented with fever, upper respiratory tract symptoms, and myalgia. He was diagnosed with COVID-19 on day 11. Lopinavir/ritonavir (LPV/r), ciclesonide, azithromycin (AZM), and ampicillin/sulbactam (ABPC/SBT) were initiated after diagnosis. He needed invasive mechanical ventilation (IMV) due to a rapid progressive respiratory failure on day 13. The ratio of arterial oxygen partial pressure to fractional inspired oxygen 
$(\mathrm{P} / \mathrm{F})$ was 136, which was classified as moderate ARDS at that time. Low-dose steroid (hydrocortisone $250 \mathrm{mg}$ /day), intravenous immunoglobulin (IVIG), and sivelestat were started after intubation. The respiratory status improved temporarily, but $\mathrm{P} / \mathrm{F}$ worsened again due to evident SOP. SPT (methylprednisolone $1000 \mathrm{mg}$ for three days) was started from day 19. After this therapy, P/F, LDH, and CT findings were improved (Figure 1. , Figure 2. ). Prednisolone (PSL) $40 \mathrm{mg}(0.5 \mathrm{mg} / \mathrm{kg} /$ day) was started after the pulse therapy, and the dose was gradually tapered. He was successful extubated on day 23, negative conversion of RT-PCR was confirmed on day 43 , and finally he was discharged without oxygen demand on day 48 . PSL was finished on day 49 .

\section{Case 2}

This is the case of a 43-year-old woman with bronchial asthma who presented with fever, cough, malaise, arthralgia, and dysgeusia. She was diagnosed with COVID-19 on day 6. LPV/r was initiated when she had pneumonia with oxygen demand on day 8. Hydroxychloroquine (HCQ), AZM, and ABPC/SBT were added on day 9 since her respiratory status worsened. She needed IMV due to a rapid progressive respiratory failure on day 10. P/F was 149, which was classified as moderate ARDS at that time. Ciclesonide, low-dose steroid (hydrocortisone $250 \mathrm{mg} /$ day), IVIG, and sivelestat were started after intubation. The respiratory status improved temporarily, but P/F worsened with evident SOP. Initially, prone position therapy (PPT) was started from day 14, and SPT (methylprednisolone $1000 \mathrm{mg}$ for three days) was initiated for SOP from day 15. After this therapy, P/F, LDH, and CT findings were improved (Figure 1. ). After the pulse therapy, PSL 30mg $(0.5 \mathrm{mg} / \mathrm{kg} /$ day $)$ was started, and the dose was gradually tapered. She was successfully extubated on day 21, negative conversion of RT-PCR was confirmed on day 27 , and finally she was discharged without oxygen demand on day 31 . PSL was finished on day 38.

\section{Case 3}

This is the case of a 68-year-old man with hypertension who presented with fever, diarrhea, and dry cough. He was diagnosed with COVID-19 on day 11. Although LPV/r was initiated after diagnosis, he manifested respiratory failure from day 12 . HCQ has been added for his treatment on day 16, ABPC/SBT on day 18, ciclesonide on day 19 , and AZM and low-dose steroid (hydrocortisone $250 \mathrm{mg} /$ day) on day 22 . He needed IMV due to a progressive respiratory failure on day 23 . P/F was 170 , which was classified as moderate ARDS at that time. IVIG, sivelestat, and PPT were started after intubation. The respiratory status improved temporarily, but $\mathrm{P} / \mathrm{F}$ worsened with evident SOP. SPT (methylprednisolone 1000mg for three days) was initiated from day 29. After this therapy, P/F, LDH, and CT findings were improved (Figure 1. ). PSL was not administered after the pulse therapy. He was successfully extubated on day 30, negative conversion of RT-PCR was confirmed on day 51, and finally he was transferred to a rehabilitation hospital due to disuse syndrome without oxygen demand on day 59.

\section{Case 4}

This is the case of a 77-year-old man with hypertension, emphysema, and old tuberculosis, who is an exsmoker and who presented with fever and sore throat. Levofloxacin and ciclesonide were administered on day 8 for bilateral pneumonia with respiratory failure. He was diagnosed with COVID-19 on day 10. He needed IMV due to a rapid progressive respiratory failure on day $11 . \mathrm{P} / \mathrm{F}$ was 170 , which was classified as moderate ARDS at that time. Although favipiravir has been initiated, low-dose steroid (hydrocortisone 250mg/day), IVIG, sivelestat, and PPT were administered after intubation. The respiratory status improved temporarily, but P/F worsened with evident SOP. SPT (methylprednisolone 1000mg for three days) was initiated from day 14. After this therapy, P/F, LDH, and CT findings were improved (Figure 1. ). PSL $30 \mathrm{mg}(0.5 \mathrm{mg} / \mathrm{kg} /$ day) was started after the pulse therapy, and the dose was gradually tapered. He was successfully extubated on day 30, negative conversion of RT-PCR was confirmed on day 24, and finally he was discharged without oxygen demand on day 30. PSL was finished on day 33.

\section{Summary of the Results for Four Cases}

SPT for SOP with respiratory failure due to COVID-19 was administered to four patients (three males, one 
female). The median age was 64.5 years (43-77). The comorbidities were hypertension in three cases, history of smoking in two cases, and bronchial asthma, emphysema in one case. The median time to diagnosis, respiratory failure, or intubation was 10.5 days (6-11), 9.5 days (8-12), or 12 days (10-23), respectively. The median findings of body temperature, lymphocyte, $\mathrm{LDH}$, and C-reactive protein on intubation were 38.5 degrees Celsius (36.8-38.6), 393.5/ $\mu \mathrm{l}$ (219-1227), 442.5IU/1 (364-599), and 15.7mg/dl (12.8-35.8), respectively. LPV/r, HCQ, favipiravir, and ciclesonide which are currently expected to have an antiviral effect were administered on day 10.5 (8-19) (median, range). IVIG and antibiotics were administered in all cases. PPT was performed in three cases. Low-dose corticosteroids were administered in all cases around the timing of intubation. SPT improved P/F, LDH, and CT findings in all cases in spite of bimodal worseness after intubation (Figure 1. , Figure 2. ). The median duration of systemic corticosteroid, intubation, ICU stay, and negative conversion of RT-PCR was 26 days (13-37), 9.5 days (8-12), 11 days (9-17), and 35 days (24-51), respectively. All cases succeeded in withdrawing from oxygen therapy, but one patient coexisted disuse symptom. No other adverse events were observed.

Discussion

This was the first study to describe the efficacy of SPT for SOP due to severe COVID-19. These cases are relatively elderly with some comorbidities, similar as that of severe previous cases ${ }^{5}$. The length of ICU stay in this study was similar as that of Zhou et $\mathrm{al}^{6}$. It was reported that the time to negative conversion of RT-PCR was 20 days in mild to severe cases ${ }^{6,7}$. However, this study had it longer than that of the previous study. It was suggested that systemic corticosteroids might delay viral shedding. Since RT-PCR tests were not performed on continuous days, the time to negative conversion of RT-PCR might be shorter in some cases. One patient coexisted disuse syndrome, which might be associated with systemic corticosteroids and bed rest for a long time. However, it was significant that all patients could survive without oxygen therapy. Therefore, SPT might be beneficial for SOP with respiratory failure. However, corticosteroids should be used in a short-term to diminish this toxicity.

In conclusion, SPT is effective for SOP with respiratory failure due to COVID-19. Although the use of systemic corticosteroids is of concern due to the delay of viral shedding or toxicity, they can be used safely by selecting optimal cases, timing, and dose. It is necessary to establish its evidence for further case accumulation and research.

\section{References}

1. Scott S, Kay FU, Suhny A, et al. Radiological Society of North America Expert Consensus Statement on reporting chest CT findings related to COVID-19. Endorsed by the Society of Thoracic Radiology, the American College of Radiology, and RSNA. J Thorac Imaging. In press 2020.

2. Bradley B, Branley HM, Egan JJ, et al. Interstitial lung disease guideline: The British Thoracic Society in collaboration with the Thoracic Society of Australia and New Zealand and the Irish Thoracic Society. Thorax . 2008;63(Suppl 5):v1-v58.

3. Russell CD, Millar JE, Baillie JK. Clinical evidence does not support corticosteroid treatment for 2019-nCoV lung injury. Lancet . 2020;395(10223):473-475.

4. Wu C, Chen X, Cai Y, et al. Risk factors associated with acute respiratory distress syndrome and death in patients with Coronavirus Disease 2019 pneumonia in Wuhan, China. JAMA Intern Med. 2020 Mar 13;e200994. Epub 2020 Mar 13.

5. Wang D, Hu B, Hu C, et al. Clinical characteristics of 138 hospitalized patients with 2019 Novel Coronavirus-infected pneumonia in Wuhan, China. JAMA. 2020;323(11):1061-1069.

6. Zhou F, Yu T, Du R, et al. Clinical course and risk factors for mortality of adult inpatients with COVID-19 in Wuhan, China: A retrospective cohort study. Lancet. 2020;395(10229):1054-1062.

7. Xiao AT, Tong YX, Gao C, Zhu L, Zhang YJ, Zhang S. Dynamic profile of RT-PCR findings from 301 COVID-19 patients in Wuhan, China: A descriptive study. J Clin Virol.2020;127:104346.

\section{Figure Legends}

Figure 1. Clinical course of $\mathrm{P} / \mathrm{F}$ (the ratio of arterial oxygen partial pressure to fractional inspired oxygen) 
and LDH (IU/l). Black triangle: the day of intubation. Black square: the day of extubation. Day 1 was set as the initiation of steroid pulse therapy.

Figure 2. Computed tomography of the chest during the clinical course in case 1. It showed bilateral peripheral ground glass opacities on day 11 (on admission). These opacities progressed to SOP, showing consolidation with traction bronchiectasis and volume loss on day 19 (the day steroid pulse therapy was started). Patient almost recovered from these findings on day 61.
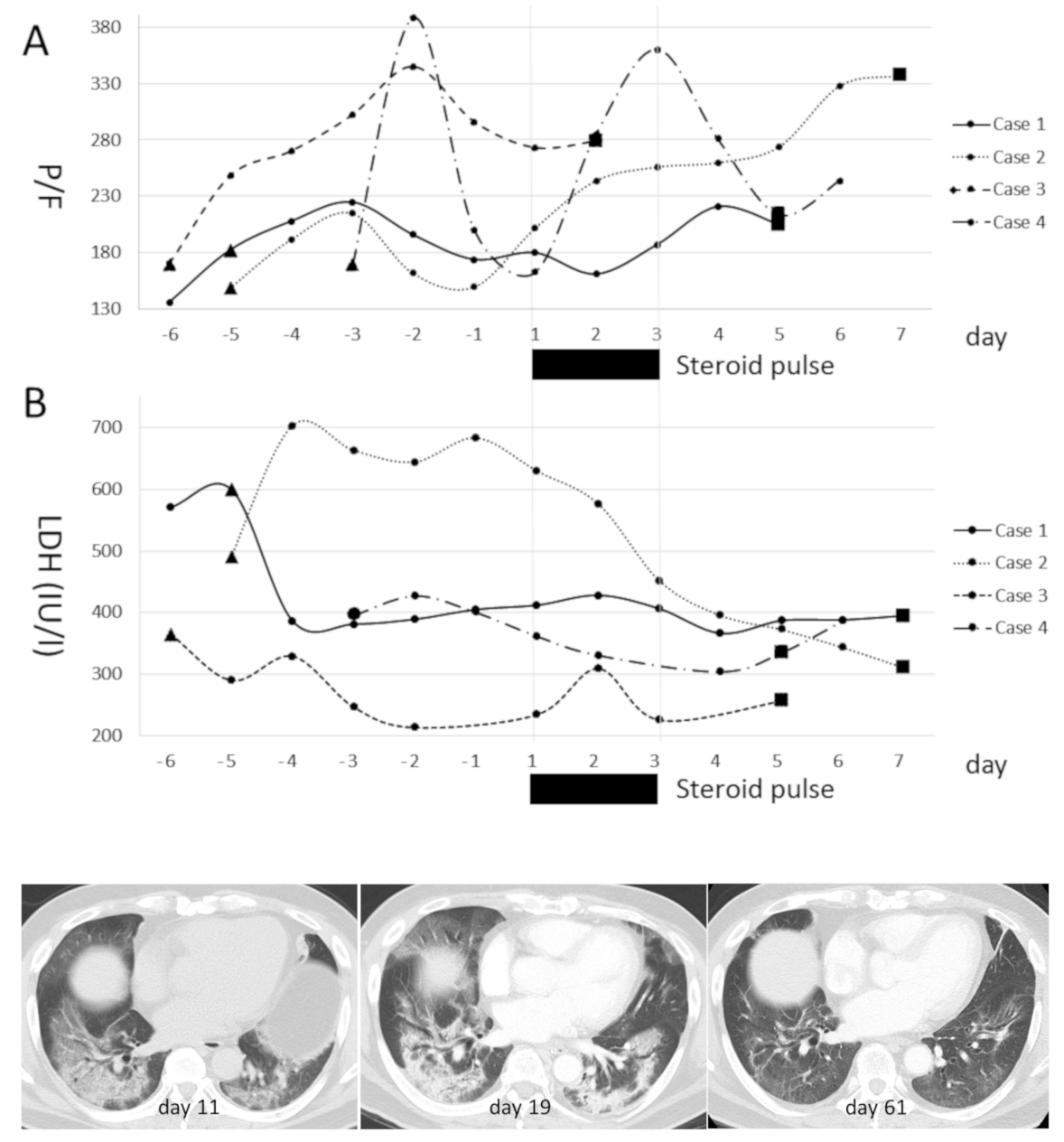